\title{
Effets génétiques et non génétiques sur le poids de toison des ovins des races Boujaâd et Sardi
}

\author{
A. Chikhi ${ }^{1}$ I. Boujenane ${ }^{2}$
}

\begin{abstract}
Mots-clés
Ovin Boujaâd - Ovin Sardi - Laine Héritabilité - Progrès génétique Maroc.
\end{abstract}

\begin{abstract}
Résumé
L'analyse a porté sur 1937 et 2005 poids de toison des ovins, respectivement des races Boujaâd et Sardi. Les données ont été collectées durant huit ans, de 1994 à 2001, dans le domaine expérimental Déroua à l'Inra. L'âge à la tonte, I'année de tonte et le sexe de l'animal ont eu des effets significatifs sur le poids de toison des ovins des deux races. Les animaux âgés de moins de 18 mois et de sexe mâle ont montré les performances les plus élevées. La répétabilité et l'héritabilité du poids de toison ont été estimées par la méthode Reml appliquée à un modèle animal. Les répétabilités estimées du poids de la toison ont été de 0,46 et 0,57 respectivement pour les ovins des races Boujaâd et Sardi, et les héritabilités ont été respectivement de 0,27 et 0,40. La régression de la valeur génétique additive de chaque animal pour le poids de toison, estimée par la méthode Blup appliquée à un modèle animal en son année de naissance a montré un progrès génétique annuel de 32 g/an pour la race Boujaâd et de 68 g/an pour la race Sardi. Il a été conclu que la sélection serait très efficace pour l'amélioration du poids de toison des ovins des races Boujaâd et Sardi.
\end{abstract}

\section{INTRODUCTION}

La production des ovins est caractérisée par trois produits principaux, à savoir la viande, le lait et la laine. Au Maroc, la priorité est accordée à la production de viande dans le but de satisfaire la demande de la population en protéines animales ; la laine est considérée comme un sous-produit de l'élevage. En effet, bien que la production de la laine soit annuellement de 36000 tonnes, elle est essentiellement destinée à la literie et à la fabrication des tapis. L'industrie textile fait rarement appel à la laine locale qu'elle considère de mauvaise qualité à cause de la forte présence de jarres dans la toison (4). Par conséquent, il est important de mettre en place un programme de sélection afin d'augmenter le poids de toison, d'améliorer la qualité de la laine et de valoriser les laines produites par les races locales (5). Ce programme de sélection, qui peut dans un premier temps s'intéresser à l'amélioration du poids de toison, nécessite pour sa réussite la connaissance des facteurs

1. Institut national de la recherche agronomique, Errachidia, Maroc.

2. Département des Productions animales, Institut agronomique et vétérinaire Hassan II, BP 6202, Rabat-Instituts, 10101 Rabat, Maroc.

Tél./fax : +212 37776420 ; e-mail : i.boujenane@iav.ac.ma

* Auteur pour la correspondance non génétiques qui affectent ce caractère et l'estimation des paramètres génétiques et phénotypiques.

La présente étude vise la détermination des principaux facteurs non génétiques qui influencent le poids de toison des ovins des races Boujaâd et Sardi, l'estimation de la répétabilité, de l'héritabilité, ainsi que du progrès génétique réalisé par sélection.

\section{MATERIEL ET METHODES}

\section{Source des données}

L'étude a porté sur l'analyse de 1937 poids de toison obtenus à partir de 427 brebis et béliers issus de 33 pères de race Boujaâd et de 2005 poids de toison de 579 brebis et béliers issus de 24 pères de race Sardi. Les poids de toison ont été collectés durant huit années, de 1994 à 2001, dans le domaine expérimental Déroua (DED) à l'Institut national de la recherche agronomique (Inra) de Béni Mellal qui est localisé à $300 \mathrm{~km}$ au sud-est de Rabat. Les brebis tondues de race Boujaâd ont eu un rang d'agnelage moyen de 3,54 (rangs de 1 à 8 ) et un âge à la tonte moyen de 48,3 mois. Celles de race Sardi ont eu un rang d'agnelage moyen de 2,98 (rangs de 1 à 8 ) et un âge à la tonte moyen de 42,8 mois. La répartition du nombre de toisons par race, par sexe et par année est présentée dans le tableau I. 


\section{Tableau I}

Répartition du nombre de données par race, par sexe et par année de tonte

\begin{tabular}{lrrrrrrr}
$\begin{array}{l}\text { Année } \\
\text { de tonte }\end{array}$ & \multicolumn{3}{c}{ Race Boujaâd } & \multicolumn{3}{c}{ Race Sardi } \\
\cline { 2 - 4 } & & & & & & \\
\cline { 6 - 7 } & Mâle & Femelle & Total & Mâle & Femelle & Total \\
1994 & - & 134 & 134 & - & 105 & 105 \\
1995 & 17 & 190 & 207 & 30 & 257 & 287 \\
1996 & 23 & 235 & 258 & 26 & 275 & 301 \\
1997 & 18 & 235 & 253 & 21 & 213 & 234 \\
1998 & 46 & 246 & 292 & 45 & 213 & 258 \\
1999 & 40 & 225 & 265 & 36 & 207 & 243 \\
2000 & 51 & 239 & 290 & 49 & 239 & 288 \\
2001 & 51 & 187 & 238 & 50 & 239 & 289 \\
Total & 247 & 1690 & 1937 & 257 & 1748 & 2005
\end{tabular}

\section{Conduite des animaux}

Les animaux des deux races étaient conduits en stabulation permanente et recevaient une alimentation à l'auge à base de foin de luzerne, de paille, d'orge grain, de maïs grain, de pulpe sèche de betterave, de tourteau de tournesol et d'un complément minéral et vitaminé. Les brebis se reproduisaient au rythme d'un agnelage par an. La lutte contrôlée était réalisée chaque année aux mois de juin et juillet et durait 60 jours. Les agnelages avaient lieu durant la période novembre - décembre. Les agneaux étaient sevrés à l'âge de 90 jours. Les animaux étaient vaccinés pour prévenir l'entérotoxémie, la clavelée et la myopathie, et ils étaient traités contre les parasites internes et externes. Des informations plus détaillées sur les races Sardi et Boujaâd et le mode de conduite du troupeau ont été rapportées par Chikhi et Boujenane $(7,8)$.

\section{Contrôles effectués}

Les animaux étaient tondus manuellement une fois par an, vers avril - mai. La tonte concernait tous les animaux du troupeau (brebis, béliers, antenaises et antenais), excepté les agneaux nés durant l'année. L'âge à la première tonte des animaux du troupeau était en moyenne de 17,5 mois. Le jour de la tonte, la toison de chaque animal était pesée, et le numéro, la date de tonte, le rang de tonte et le sexe de l'animal étaient enregistrés.

\section{Analyses statistiques}

L'analyse a été faite séparément pour chaque race. La détermination des effets des facteurs non génétiques qui ont influencé le poids de toison a été faite par la méthode des moindres carrés en utilisant la procédure des modèles linéaires généralisés (GLM) du logiciel SAS (21). Le modèle final incluait les effets fixes de l'âge à la tonte $(7$ niveaux : âge $<18$ mois, $18 \leq$ âge $<30$ mois, $30 \leq$ âge $<42$ mois, $42 \leq$ âge $<54$ mois, $54 \leq$ âge $<66$ mois, $66 \leq$ âge $<78$ mois et âge $\geq 78$ mois), le sexe ( 2 niveaux : mâle et femelle), l'année de tonte (8 niveaux : 1994 ... 2001) et l'interaction entre l'âge à la tonte et le sexe.

L'estimation des composantes de la variance a été faite en utilisant la méthode de vraisemblance maximale restreinte (Reml) à l'aide du programme Mtdfreml (3). Le modèle animal utilisé a inclus les effets fixes (âge à la tonte, sexe, année de tonte), l'effet aléatoire de l'animal et l'effet aléatoire de l'environnement permanent. La convergence a été considérée comme atteinte lorsque la variance du simplex a été inférieure à $10^{-8}$. La représentation matricielle du modèle a été comme suit :

$\mathrm{y}=\mathrm{Xb}+\mathrm{Za}+\mathrm{We}_{\mathrm{p}}+\mathrm{e}$

où $\mathrm{y}$ est le vecteur des observations, $\mathrm{X}$ la matrice d'incidence qui relie les observations aux effets fixes, b le vecteur des effets fixes, $\mathrm{Z}$ la matrice d'incidence qui relie les observations aux valeurs génétiques additives, a le vecteur des valeurs génétiques additives $\sim\left(0, A \sigma^{2}{ }_{a}\right), W$ la matrice d'incidence qui relie les observations aux effets de l'environnement permanent, $\mathrm{e}_{\mathrm{p}}$ le vecteur de l'environnement permanent $\sim\left(0, \mathrm{I}_{c} \sigma_{c}^{2}\right)$ et e le vecteur des résiduelles $\sim(0$, $\mathrm{I}_{\mathrm{n}} \sigma_{\mathrm{e}}^{2}$ ). En outre, A est la matrice des relations génétiques additives entre les animaux, $I_{c}$ une matrice unité de dimension égale au nombre d'animaux, $\mathrm{I}_{\mathrm{n}}$ une matrice unité de dimension égale au nombre d'observations, $\sigma_{a}^{2}$ la variance génétique additive, $\sigma_{c}^{2}$ la variance de l'environnement permanent et $\sigma_{\mathrm{e}}^{2}$ la variance résiduelle.

La répétabilité ( $r$ ) du poids de toison a été estimée par $r=\frac{\sigma_{\mathrm{a}}^{2}+\sigma_{\mathrm{e}}^{2}}{\sigma_{\mathrm{p}}^{2}}$ et son héritabilité $\left(h^{2}\right)$ par $h^{2}=\frac{\sigma_{a}^{2}}{\sigma_{p}^{2}}$, où $\sigma_{p}^{2}$ est la variance phé-
notypique totale.

Les valeurs génétiques de tous les animaux (individus, pères et mères) présents dans le fichier de données analysées ont été estimées en utilisant le modèle précédent. Le progrès génétique annuel réalisé a été calculé par la régression de la valeur génétique additive estimée de chaque animal en son année de naissance.

\section{RESULTATS ET DISCUSSION}

La moyenne arithmétique du poids de toison des ovins de race Boujaâd a été de $3,41 \mathrm{~kg}$, avec un coefficient de variation de 23,1 p. 100. Celle du poids de toison des ovins de race Sardi a été de $2,00 \mathrm{~kg}$, avec un coefficient de variation de 36,8 p. 100. Les moyennes ajustées du poids de toison des ovins des races Boujaâd et Sardi par facteur de variation sont rapportées dans le tableau II.

\section{Effet de l'âge à la tonte}

L'âge à la tonte a eu un effet significatif sur le poids de toison des ovins des races Boujaâd et Sardi. Le poids de toison le plus élevé a été enregistré chez les ovins âgés de moins de 18 mois, c'est-à-dire chez les animaux tondus pour la première fois. Le plus faible poids a été obtenu chez ceux âgés de plus de 78 mois. Les différences entre les classes extrêmes ont été de 1,89 kg chez les ovins Boujaâd et $1,45 \mathrm{~kg}$ chez les ovins Sardi. Ces résultats peuvent être expliqués par la diminution du nombre et de la densité des fibres avec l'âge des animaux. Plusieurs auteurs ont rapporté que l'âge à la tonte affecte le poids de la toison. Eikje (10) rapporte que les brebis âgées de deux ans (première tonte) des cinq races étudiées produisent des toisons de 0,3 à $0,5 \mathrm{~kg}$ plus lourdes que celles des autres groupes d'âge. Le poids de toison des ovins des races Sardi, Timahdite et de leurs races croisées est de 2,21 kg à un âge inférieur à 24 mois (première tonte) et de $1,55 \mathrm{~kg}$ à un âge de plus de 60 mois (11).

\section{Effet du sexe}

Le sexe des animaux des races Boujaâd et Sardi a influencé de manière très significative le poids de toison $(p<0,001)$. Les mâles ont produit des toisons plus lourdes que les femelles. Cette supériorité a été de $0,54 \mathrm{~kg}$ chez la race Boujaâd et de $0,27 \mathrm{~kg}$ chez la race Sardi. Ces résultats confirment ceux de Basuthakur et coll. (2) qui trouvent 


\section{Tableau II}

Moyennes ajustées \pm erreurs types du poids de toison des ovins Boujaâd et Sardi

\begin{tabular}{|c|c|c|c|c|}
\hline \multirow[t]{2}{*}{ Facteur de variation } & \multicolumn{2}{|c|}{ Race Boujaâd } & \multicolumn{2}{|c|}{ Race Sardi } \\
\hline & Nb. & Moyenne ajustée (kg) & Nb. & Moyenne ajustée (kg) \\
\hline Age à la tonte (mois) & & $* * *$ & & * \\
\hline$<18$ & 343 & $4,53 \pm 0,05^{a}$ & 494 & $2,78 \pm 0,04^{a}$ \\
\hline $19 \leq$ âge $<30$ & 365 & $3,83 \pm 0,05 b$ & 403 & $2,19 \pm 0,05 b$ \\
\hline $30 \leq$ âge < 42 & 265 & $3,54 \pm 0,08^{d}$ & 285 & $2,06 \pm 0,07^{c d}$ \\
\hline $42 \leq$ âge $<54$ & 310 & $3,36 \pm 0,07^{d}$ & 312 & $1,91 \pm 0,08^{d}$ \\
\hline $54 \leq$ âge $<66$ & 212 & $3,01 \pm 0,10^{c}$ & 215 & $1,80 \pm 0,10^{e}$ \\
\hline $66 \leq$ âge $<78$ & 188 & $3,16 \pm 0,18^{\mathrm{cd}}$ & 149 & $1,42 \pm 0,37$ се \\
\hline Age $\geq 78$ & 254 & $2,64 \pm 0,23^{c}$ & 147 & $1,33 \pm 0,19 \mathrm{e}$ \\
\hline Sexe & & $* * *$ & & $* * *$ \\
\hline Mâle & 247 & $3,71 \pm 0,10^{a}$ & 257 & $2,06 \pm 0,12^{a}$ \\
\hline Femelle & 1690 & $3,17 \pm 0,02^{b}$ & 1748 & $1,79 \pm 0,02 b$ \\
\hline Année de tonte & & $* * *$ & & $* * *$ \\
\hline 1994 & 134 & $2,57 \pm 0,09 a$ & 105 & $1,59 \pm 0,10^{a}$ \\
\hline 1995 & 207 & $3,56 \pm 0,08$ ef & 287 & $1,69 \pm 0,08$ ae \\
\hline 1996 & 258 & $4,06 \pm 0,07 b$ & 301 & $2,08 \pm 0,08 b$ \\
\hline 1997 & 253 & $3,25 \pm 0,07^{c}$ & 234 & $1,93 \pm 0,08$ се \\
\hline 1998 & 292 & $3,91 \pm 0,07 \mathrm{~g}$ & 258 & $1,97 \pm 0,08 b c$ \\
\hline 1999 & 265 & $3,46 \pm 0,07^{e}$ & 243 & $2,27 \pm 0,08^{d}$ \\
\hline 2000 & 290 & $3,70 \pm 0,06^{f}$ & 288 & $2,07 \pm 0,07^{b}$ \\
\hline 2001 & 238 & $3,01 \pm 0,07 d$ & 289 & $1,81 \pm 0,08 e^{e}$ \\
\hline
\end{tabular}

que les poids de toison des mâles sont plus élevés que ceux des femelles. La supériorité du poids de toison des mâles peut être expliquée par leur taille plus grande que celle des brebis et aussi par une différence d'origine hormonale (13). De plus chez, les femelles la croissance de la laine est réduite de 20 à 40 p. 100 durant la dernière période de gravidité et de 30 p. 100 pendant la période de lactation (9).

\section{Effet de l'année de tonte}

L'année de tonte a eu un effet très significatif sur le poids de toison des brebis et des béliers des races Boujaâd et Sardi $(\mathrm{p}<0,001)$. Ces résultats sont en accord avec ceux d'Aboul-Naga et Afifi (1) et de Sidwell et coll. (22) qui rapportent que l'année de tonte affecte de manière significative le poids de toison. Chez les ovins Boujaâd et Sardi, le poids de toison le plus élevé a été enregistré en 1996, alors que le plus faible a été obtenu en 1994. Ces variations peuvent être expliquées par les changements au niveau des rations alimentaires, et au niveau de la fertilité et de la prolificité des brebis.

\section{Effet de l'interaction entre l'âge et le sexe}

L'interaction entre l'âge à la tonte et le sexe de l'animal a eu un effet significatif sur le poids de toison des races Boujaâd et Sardi. En effet, le sexe a agi différemment sur ce caractère en fonction de l'âge à la tonte. La différence de poids de toison entre les mâles et les femelles a été élevée chez les animaux dont l'âge était de moins de 18 mois et de 18 à 30 mois, et elle a été faible chez les animaux des autres classes d'âge.

\section{Répétabilité et héritabilité}

Les estimations des composantes de la variance, ainsi que la répétabilité et l'héritabilité du poids de toison par race sont rapportées dans le tableau III. La répétabilité du poids de toison des ovins de race Sardi $(0,57)$ a été plus élevée que celle des ovins de race Boujaâd $(0,46)$. Ces estimations moyennement élevées indiquent que la connaissance d'un seul poids de toison est suffisante pour porter un bon jugement sur les animaux de race Boujaâd et surtout de race Sardi. Toutefois, ces estimations ont été inférieures à celles obtenues par plusieurs auteurs $(12,14,15,17,18,20)$ sur les races Béni Guil, Columbia, Polypay, Rambouillet et Targhee, qui ont varié entre 0,58 et 0,70 .

L'héritabilité du poids de toison a été plus élevée chez les ovins Sardi que chez les ovins Boujaâd. Elle a été de 0,40 chez la race Sardi et de 0,27 chez la race Boujaâd. La sélection sur le poids de toison pourrait aboutir à un gain génétique appréciable chez les deux races, mais il sera plus rapide chez la race Sardi que chez la race Boujaâd. Les héritabilités estimées dans la présente étude ont été inférieures à celles rapportées chez plusieurs races (Béni Guil, Rambouillet, Polypay, Targhee, Columbia et West White Faced), qui ont varié de 0,52 à $0,62(6,14,15,16,17$, 18, 20). Mais, elles ont été supérieures à l'héritabilité moyenne du poids de toison des races à viande $(0,19)$ et l'héritabilité de la race Sardi a été plus élevée que les valeurs moyennes obtenues aussi bien chez les races lainières $(0,34)$ que chez les races mixtes $(0,34)(12)$. 
Tableau III

Estimation des composantes de la variance du poids de toison des ovins Boujaâd et Sardi

\section{Paramètres}

\begin{tabular}{|c|c|c|c|c|c|c|c|c|}
\hline Race & $\sigma_{p}^{2}$ & $\sigma_{\mathrm{a}}^{2}$ & $\sigma_{e_{p}}^{2}$ & $\sigma_{\mathrm{e}}^{2}$ & $\mathrm{~h}^{2}$ & $\mathrm{r}$ & $c^{2}=\frac{\sigma_{e_{p}}^{2}}{\sigma_{p}^{2}}$ & $\mathrm{t}^{2}=\frac{\sigma_{\mathrm{e}}^{2}}{\sigma_{\mathrm{p}}^{2}}$ \\
\hline Boujaâd & 0,696 & 0,188 & 0,133 & 0,375 & 0,27 & 0,46 & 0,19 & 0,54 \\
\hline Sardi & 0,540 & 0,216 & 0,094 & 0,230 & 0,40 & 0,57 & 0,17 & 0,43 \\
\hline
\end{tabular}

\section{Estimation du progrès génétique réalisé}

Les moyennes des valeurs génétiques additives pour le poids de toison ont augmenté d'une année de naissance à l'autre de façon nette et progressive chez les ovins de race Boujaâd, et de manière relativement lente chez les ovins de race Sardi. Elles ont varié de presque 0 chez les animaux nés en 1992, à approximativement 0,38 kg en $1998 \mathrm{chez}$ les ovins Boujaâd, et à 0,69 kg en $1993 \mathrm{chez}$ les ovins Sardi (figures 1 et 2). Le progrès génétique annuel réalisé sur le poids de toison a été en moyenne de $32 \mathrm{~g} / \mathrm{an}$ pour la race Boujaâd et de $68 \mathrm{~g} / \mathrm{an}$ pour la race Sardi. Ces gains génétiques élevés indiquent que l'amélioration du poids de toison chez les races Sardi et Boujaâd peut être efficace et rapide. Les progrès génétiques annuels réalisés chez les races Boujaâd et Sardi ont été supérieurs au progrès génétique presque négligeable observé chez les races Columbia et Targhee $(14,15)$, et à la réponse corrélative de l'ordre de $13 \mathrm{~g} / \mathrm{an}$ obtenue chez les races Border Leicester et Coopworth lorsque la sélection est pratiquée sur la croissance (19).

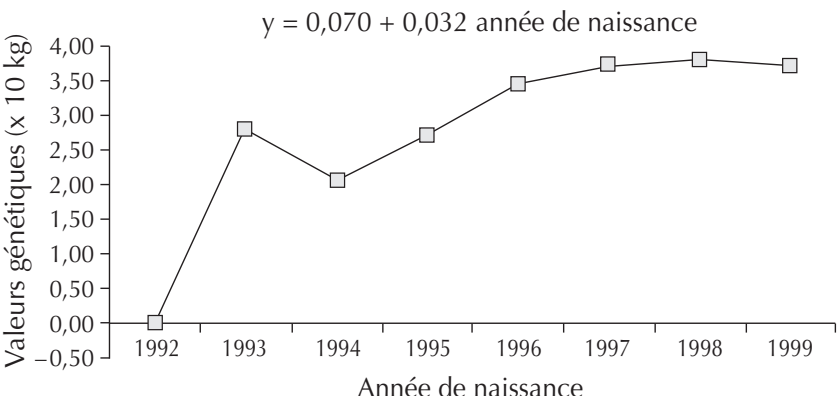

Figure 1 : évolution des valeurs génétiques additives des ovins de race Boujaâd pour les poids de toison selon l'année de naissance.

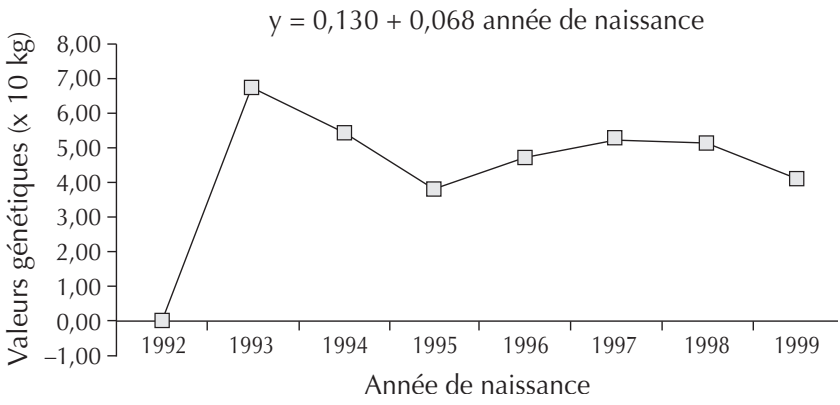

Figure 2 : évolution des valeurs génétiques additives des ovins de race Sardi pour les poids de toison selon l'année de naissance.

\section{CONCLUSION}

Le poids de toison des ovins Boujaâd et Sardi a été significativement influencé par l'âge à la tonte, le sexe de l'animal et l'année de tonte. Tout programme de sélection visant l'amélioration de ce caractère doit prendre en considération les effets de ces facteurs lors de l'évaluation génétique des animaux. L'héritabilité élevée du poids de toison des ovins Sardi et Boujaâd indique que la sélection peut être efficace pour l'amélioration de ce caractère, comme en témoignent les progrès génétiques élevés qui ont été réalisés. Néanmoins, la sélection pour l'augmentation du poids de toison tout seul résulte d'habitude en une laine grossière et peu fine, comme en témoignent les corrélations génétiques positives entre le poids de la toison et le diamètre de la fibre de laine $(14,15)$. Afin d'améliorer à la fois la quantité et la qualité de la laine, et de répondre ainsi à la demande des utilisateurs, le programme de sélection doit inclure à la fois le poids de toison et le diamètre de la fibre. Les deux caractères seront améliorés, mais le progrès génétique réalisé sur chacun d'entre eux sera plus faible que celui qui serait obtenu si la sélection était faite sur un seul caractère à la fois.

\section{Remerciements}

Les auteurs adressent leurs vifs remerciements à M. L. Haounou, technicien au domaine expérimental Déroua, pour sa collaboration technique.

\section{BIBLIOGRAPHIE}

1. ABOUL-NAGA A.M., AFIFI E.A., 1977. Environment and genetic factors affecting wool production from sub-tropical coarse wool sheep. J. Agric. Sci. Camb., 88: 443-447.

2. BASUTHAKUR A.K., BURFENING P.J., VAN HORN J.L., BLACKWELL R.L., 1973. A study of some aspects of lifetime production in Targhee and Columbia sheep. J. Anim. Sci., 36: 813-820.

3. BOLDMAN K.G., KRIESE L.A., VAN VLECK L.D., KACHMAN S.D., 1993. A manual for MTDFREML, a set of programs to obtain estimates of variances and covariances. Washington, DC, USA, USDA, 120 p.

4. BOUJENANE I., 1999. Les ressources génétiques ovines au Maroc. Rabat, Maroc, Actes Editions, 136 p.

5. BOURFIA M., LAIDOUNI M.S., EL HMAMSI M.M., 1987. Caractéristiques des toisons des principales races ovines locales. In $: 17^{\text {es }}$ Journées de l'Association nationale pour la production animale, Rabat, Maroc

6. BROMLEY C.M., SNOWDER G.D., VAN VLECK L.D., 2000. Genetic parameters among weight, prolificacy, and wool traits of Columbia, Polypay, Rambouillet and Targhee sheep. J. Anim. Sci., 78: 846-858.

7. CHIKHI A., BOUJENANE I., 2003. Performances de reproduction et de production des ovins de race Boujaâd au Maroc. Revue Elev. Méd. vét. Pays trop., $\mathbf{5 6}:$ :83-88. 
8. CHIKHI A., BOUJENANE I., 2003. Caractérisation zootechnique des ovins de race Sardi au Maroc. Revue Elev. Méd. vét. Pays trop., 56 : 187-192.

9. DONEY J.M., 1983. Factors affecting the production and quality of wool. In: Haresign W., Ed., Sheep production. London, UK, Butterworths, p. 537-544.

10. EIKJE E.D., 1971. Studies on sheep production records. II. Effect of environmental factors on fertility, fleece and body weight of ewes. Acta Agric. Scand., 21: 64-68.

11. EL FADILI M., 1997. Intensification de la production ovine par le croisement. Mémoire Ingénieur en chef, Inra, Rabat, Maroc.

12. FOGARTY N.M., 1995. Genetic parameters for live weight, fat and muscle measurements, wool production and reproduction in sheep: A review. Anim. Breed. Abstr., 63: 101-143.

13. GUIRSIS R.A., AFIFI E.A., GALAL E.S.E., 1982. Estimates of genetic and phenotypic parameters of some weight and fleece traits in coarse wool breed of sheep. J. Agric. Sci. Camb., 99: 277-285

14. HANFORD K.J., VAN VLECK L.D., SNOWDER G.D., 2002. Estimates of genetic parameters and genetic change for reproduction, weight and wool characteristics of Columbia sheep. J. Anim. Sci., 80: 3086-3098.

15. HANFORD K.J., VAN VLECK L.D., SNOWDER G.D., 2003. Estimates of genetic parameters and genetic change for reproduction, weight and wool characteristics of Targhee sheep. J. Anim. Sci., 81: 630-640.
16. IMAN N.Y., JOHNSON C.L., RUSSEL L.D., STOBART R.H., 1992. Estimation of genetic parameters estimates for wool fiber measures. J. Anim. Sci., 70: 1110-1115.

17. LEE J.W., WALDRON D.F., VAN VLECK L.D., 2000. Parameter estimates for greasy fleece weight of Rambouillet sheep at different ages. J. Anim. Sci., 78: 2108-2112.

18. MHARCHI A., 1991. Analyse génétique des performances de reproduction et de production des ovins de race Beni Guil à la ferme de sélection ovine d'Oued Isly à Oujda. Mémoire $3^{\mathrm{e}}$ cycle Agronomie, IAV Hassan II, Rabat, Maroc, 180 p.

19. NSOSO S.J., YOUNG M.J., BEATSON P.R., 1999. Correlated responses in greasy fleece weight trait in Border Leicester and Coopworth sheep breeds selected for lean tissue growth rate. Small Rumin. Res., 34 149-154.

20. OKUT H., BROMLEY C.M., VAN VLECK L.D., SNOWDER G.D., 1999. Genotypic expression at different ages. II. Wool traits for sheep. J Anim. Sci., 77: 2366-2371.

21.SAS/STAT, 1996. User's Guide, vers. 6.12. Cary, NC, USA, SAS Institute.

22. SIDWELL G.M., WILSON R.L., HOURIHAN M.E., 1971. Production in some pure breeds of sheep and their crosses. IV. Effect of crossbreeding on wool production. J. Anim. Sci., 32: 1099-1102.

Accepté le 19.09.2006

\section{Summary}

Chikhi A., Boujenane I. Genetic and non-Genetic Effects on the Fleece Weight of Boujaâd and Sardi Sheep Breeds

The analysis concerned 1937 and 2005 fleece weights of Boujaâd and Sardi sheep breeds, respectively. Data were collected during eight years from 1994 to 2001 at Deroua Experimental Station of INRA. Age at shearing, year of shearing and the animal sex had significant effects on the fleece weight of sheep of both breeds. Males under the age of 18 months showed the highest performance. Repeatability and heritability of fleece weights were estimated with the REML method applied to an animal model. Repeatability estimates for fleece weight were 0.46 and 0.57 for Boujaâd and Sardi, respectively, and heritability estimates were 0.27 and 0.40 , respectively. The regression of the additive genetic value of each animal for fleece weight, estimated by the BLUP animal model, on its birth year, showed an annual genetic progress of 32 g/year for Boujaâd and $68 \mathrm{~g}$ /year for Sardi. It was concluded that selection would be very efficient to improve the fleece weights of Boujaâd and Sardi breeds.

Keywords: Boujaâd sheep - Sardi sheep - Wool - Heritability Genetic progress - Morocco.

\section{Resumen}

Chikhi A., Boujenane I. Efectos genéticos y no genéticos sobre el peso del vellocino de los ovinos de razas Boujaâd y Sardi

El análisis se centró sobre 1937 y 2005 pesos de vellocino de ovinos, de las razas Boujaâd y Sardi respectivamente. Los datos fueron colectados durante ocho años, de 1994 a 2001, en el dominio experimental Deroua del Inra. La edad de la esquila, el año de la esquila y el sexo del animal tuvieron efectos significativos sobre el peso del vellocino de los ovinos de las dos razas. Los animales de menos de 18 meses y de sexo macho mostraron los rendimientos más elevados. La repetibilidad y la heredabilidad del peso del vellocino se estimaron mediante el método REML, aplicado al modelo animal. Las repetibilidades estimadas del peso del vellocino fueron de 0,46 y 0,57 para los ovinos de razas Boujaâd y Sardi respectivamente, las heredabilidades fueron respectivamente de 0,27 y 0,40. La regresión del valor genético aditivo de cada animal para el peso del vellocino, estimado mediante el método de BLUP aplicado a un modelo animal, sobre su año de nacimiento, mostró un progreso genético anual de 32 g/año para la raza Boujaâd y de 68g/año para la raza Sardi. Se concluyõ que la selección sería eficaz para el mejoramiento del peso del vellocino de las razas Boujaâd y Sardi.

Palabras clave: Ovino Boujaâd - Ovino Sardi - Lana Heredabilidad - Mejora genética - Marruecos. 